Uptake of oral anticoagulants for stroke prevention in patients with atrial fibrillation in a single Clinical Commissioning Group in England without restrictions to their use

\title{
Uptake of oral anticoagulants for stroke prevention in atrial fibrillation
}

\author{
Authors \\ Medlinskiene Kristina ${ }^{1,2}$, Fay Matthew ${ }^{3}$, Petty Duncan ${ }^{1}$ \\ ${ }^{1}$ School of Pharmacy and Medical Sciences, University of Bradford, Bradford, United Kingdom \\ ${ }^{2}$ Medicine Management and Pharmacy Services, Leeds Teaching Hospitals NHS Trust, Leeds United Kingdom \\ ${ }^{3}$ The Willows Medical Centre, Queensbury, United Kingdom \\ ${ }^{1}$ k.medlinskiene1@bradford.ac.uk, ORCHID ID: 0000-0002-6721-3399, School of Pharmacy and Medical \\ Sciences, University of Bradford, Richmond road, Bradford, United Kingdom, BD7 1DP.
}

\begin{abstract}
Background and Objective

In England, the uptake of direct oral anticoagulants (DOAC) for stroke prevention in atrial fibrillation has been slow and varied across different Clinical Commissioning Groups (CCGs). This study aimed to profile the prescribing of oral anticoagulants for stroke prevention in patients with atrial fibrillation over three years in a CCG without restrictions to DOACs use to understand more about organisational and/or individual barriers to the early uptake of DOACs.
\end{abstract}

\section{Methods}

Data were collected from nine general practices between 01/04/2012 and 31/03/2015 of patients who were initiated on the oral anticoagulant therapy. Data were analysed descriptively and with independent Student's $t$ test and Chi-square test to explore if there was an association between type of oral anticoagulant initiated and sex, age, type of prescriber, and prior aspirin use.

\section{Results}

The early uptake of DOACs significantly increased over the study period ( $\mathrm{p}<0.0001$; medium size effect $\varphi \mathrm{c}=0.372$ ). There was no statistically significant difference between gender or age and type of oral anticoagulant initiated. Primary care prescribers were responsible for initiating the majority of oral anticoagulants (71\%; $\mathrm{N}=257)$ and driving the use of DOACs $(72 \%, \mathrm{~N}=71)$. Patients switched from aspirin to an oral anticoagulant were more likely to be initiated on warfarin than DOAC.

\section{Conclusions}

The early use of DOACs, in a CCG without restrictions to their use, was embraced by primary care prescribers in this particular CCG.

\section{Key Points}

The use of direct oral anticoagulants (DOACs) significantly increased over three years in the studied Clinical Commissioning Group (CCG) in England.

The majority of oral anticoagulants were initiated by primary care prescribers, who also were driving the early uptake of DOACs in the studied CCG. 


\section{Introduction}

The optimal use of oral anticoagulants (OAC) in patients with atrial fibrillation (AF) can reduce the five-fold increased risk of stroke and thus bring benefits to both patients and health and social care systems [1-4]. The use of antiplatelet monotherapy (e.g. aspirin) is now discouraged as much greater risk reduction is achieved with OAC therapy [5]. Historically, warfarin was the only choice of OAC before the introduction of direct oral anticoagulants (DOACs). Although DOACs have their disadvantages, they were shown to be as effective as warfarin and overcome some disadvantages of warfarin such as no need of regular coagulation monitoring, simpler dosing regimens, and fewer drug or food interactions [5-7].

In England, DOACs have been recommended by National Institute for Health and Care Excellence (NICE) for stroke prevention in non-valvular AF since 2012 (dabigatran in March 2012; rivaroxaban in May 2012; apixaban in February 2013; edoxaban in September 2015) but their uptake has been slow [8]. The uptake was expected to reach $35 \%$ of all OAC prescribing with the release of the updated national guideline for the management of AF in 2014 but only 16.5\% was achieved in 2015 [8-10]. Additionally, there was a high level of unexplained variation across different Clinical Commissioning Groups (CCGs), ranging from 4\% to $70 \%$ of all OAC prescribing [8]. Potentially due to significantly higher unit costs some CCGs managed the introduction of DOACs by reserving them after the trial with warfarin $[8,11]$. In some CCGs, the anticoagulation service was commissioned to secondary care and thus limiting initiation of DOACs to secondary care prescribers [12]. Restricted use of DOACs has also been observed in treatment centres across Europe $[11,13]$.

Little is known about impact of such restrictions on early DOAC use and consequently which type of prescriber initiates the therapy, including reasons for selecting warfarin or DOAC. This study aimed to profile the prescribing of OAC for stroke prevention in patients with AF over three years in a CCG without restrictions to DOAC use to understand more about organisational and/or individual barriers to utilizing DOACs.

\section{Methods}

\subsection{Study design and setting}

This retrospective study used primary care prescribing data from 9 General Practitioner (GP) practices in Bradford Districts CCG, England. The GP practices had list sizes of up to 10,000 patients and were using TPPSystmOne clinical software. The selected CCG comprised of 39 GP practices, serving a local population of over 300,000 patients. The anticoagulation services were provided by any qualified provider (primary or secondary care). Data collected covered a period of 3 years (01/04/2012-31/03/2013, 01/04/2013-31/03/2014, and 01/04/2014-31/03/2015).

\subsection{Study population}

Adult patients prescribed OAC between 01/04/2012 and 31/03/2015 for stroke prevention in AF were included. OACs were warfarin and DOACs (apixaban, dabigatran, rivaroxaban). The DOAC edoxaban was licensed and approved by NICE after the study period and thus was not included.

\subsection{Data collection and analysis}

Practice pharmacists ran a pre-defined search on SystmOne to identify all patients who were initiated OAC therapy from 01/04/2012 to 31/03/2015. Variables to investigate included age, sex, and type of OAC prescribed. The practice pharmacist also manually searched patients' records to identify: indication for the prescribed OAC; who initiated or recommended prescribing it; reason for prescribing DOAC rather than warfarin or vice versa; and if the patient was on aspirin prior to OAC therapy. Data were anonymised and entered on a pre-designed Excel template before being sent to the research team for analysis.

Descriptive analysis was conducted and independent Student's $t$ tests were used to compare continuous variables and Chi-square tests were used to test differences in categorical variables. We explored if there was an association between type of OAC initiated and sex, age, type of prescriber, and prior aspirin use. All statistical analyses were undertaken using SPSS 24 software program.

\section{Results}

\subsection{Patient characteristics}


Five hundred and sixty patients were prescribed OAC. One hundred and thirty-six patients were excluded due to non-AF indication (venous thrombosis $\mathrm{N}=113$; mechanical valve $\mathrm{N}=6$; unknown $\mathrm{N}=17$ ). Out of 424 patients with AF indication, 360 patients were new users to the therapy and 64 patients were anticoagulated before the study period.

Characteristics of new patients $(\mathrm{N}=360)$ initiated on OAC therapy are summarised in Table 1. Although overall more males were anticoagulated, the proportion of females being anticoagulated increased from $43 \%$ to $55 \%$ during the study period (Table 1). There was no statistically significant difference between sex or age and type of OAC initiated $(\mathrm{p}=0.813$ and $\mathrm{p}=0.263)$.

\subsection{OAC prescribing}

The majority of new patients were initiated on warfarin $(73 \%, \mathrm{~N}=262)$ rather than DOAC $(27 \%, \mathrm{~N}=98)(\mathrm{Table}$ 1). However, initiation of DOACs to patients new to OAC therapy significantly increased over time $(\mathrm{p}<0.0001$; medium size effect $\varphi c=0.372$ ).

There was no statistically significant difference between the type of prescriber (secondary or primary care) and the type of OAC initiated to new patients $(\mathrm{p}=0.927)$. Nevertheless, the majority of OAC were initiated by primary care prescribers $(71 \%$; $\mathrm{N}=257)$, who tended to select warfarin $(72 \%, \mathrm{~N}=186)$ over DOACs $(28 \%$, $\mathrm{N}=71)$. GPs $(92 \% ; \mathrm{N}=237)$ were almost exclusively responsible for initiating OAC therapy in primary care. A small proportion was initiated by practice pharmacists $(7 \% ; \mathrm{N}=18)$, nurse practitioners $(<1 \% ; \mathrm{N}=1)$, or other $(<1 \% ; \mathrm{N}=1)$.

Secondary care prescribers accounted for initiating therapy in $27 \%(\mathrm{~N}=98)$ new patients. They also tended to select warfarin $(73 \%, \mathrm{~N}=72)$ over DOAC $(27 \%, \mathrm{~N}=26)$. Cardiology consultants $(44 \%$; $\mathrm{N}=43)$ were the main prescribers, followed by elderly care consultants $(25 \% ; \mathrm{N}=24)$, unspecified speciality consultants $(21 \% ; \mathrm{N}=21)$, stroke consultants $(7 \% ; \mathrm{N}=7)$, a general medicine consultant $(1 \% ; \mathrm{N}=1)$, a cardiology surgeon $(1 \% ; \mathrm{N}=1)$, a heart failure nurse $(1 \%, \mathrm{~N}=1)$. Prescriber data were missing in $5(1 \%)$ cases.

Reasons for selecting warfarin over DOAC or vice versa were poorly documented. Only in $2 \%(\mathrm{~N}=4)$ cases of patients started on warfarin had a documented reason, which was a patient's choice. In more cases $(49 \%, \mathrm{~N}=48)$ information was documented for selecting DOAC over warfarin. Stated reasons included patient's choice (42\%; $\mathrm{N}=41)$, unable to attend warfarin clinics $(4 \% ; \mathrm{N}=4)$, needle-stick phobia $(1 \% ; \mathrm{N}=1)$, and drug interaction with warfarin $(1 \% ; \mathrm{N}=1)$.

\subsection{Switching from aspirin to $\mathrm{OAC}$}

During the study period 39\% $(\mathrm{N}=140)$ patients new to OAC therapy were switched from aspirin. There was no statistically significant difference on which OAC was initiated in these patients $(\mathrm{p}=0.084)$. However, majority of patients were switched to warfarin $(78 \%, \mathrm{~N}=109)$. Primary care prescribers switched majority of patients $(76 \%$, $\mathrm{N}=106)$, which was mainly done by GPs $(89 \% ; \mathrm{N}=94)$ and in small number of cases by practice pharmacists $(10 \% ; \mathrm{N}=11)$ or other $(1 \% ; \mathrm{N}=1)$.

\section{Discussion}

In the CCG without restrictions to DOACs use, the initiation of DOACs significantly increased over the threeyear period and majority of OACs, including DOACs, were initiated by primary care prescribers. The observed increased use of DOACs correlated with the national prescribing trend of DOACs in the UK [14,15] and was likely influenced by guideline changes (e.g. NICE, European Society of Cardiology), changes in Quality and Outcome Framework (QOF) incentives for GPs, local and national initiatives to improve anticoagulation in patients with AF, and promotion associated with DOACs use $[9,15,16]$. This CCG was (and remained) well above the average use of DOACs in the UK [17], suggesting that no restrictions to DOAC use alongside other enablers contributed positively to the uptake of DOACs.

The main strength of this study was the use of patients' routine data, and review of patients' primary care medical records. This allowed us to collect information otherwise unavailable on prescribing databases (e.g. who initiated the therapy, reasons for choosing a particular OAC) and gain greater insight into early DOAC prescribing. However, several limitations should be taken into account. The data used in the study was collected from GP practices from a single CCG, thus our findings may not be generalisable. Lack of diagnostic information limited data analysis and thus insight into appropriateness of OAC use. 
Evidence about which prescribers initiate OAC therapy (i.e. primary or secondary care prescriber) is needed to promote the optimal and rational use of DOACs but is currently limited. Patients with AF are predominantly managed in primary care setting and thus primary care prescribers are in the best position to identify patients with undiagnosed $\mathrm{AF}$ and undertreated with OAC therapy. Our study showed that $71 \%$ of new OAC prescriptions were solely initiated by primary care prescribers, predominantly GPs. In contrast, a Danish OAC utilization study using the prescribing data (August 2011 to June 2013) showed that hospital prescribers initiated vitamin $\mathrm{K}$ antagonists and DOACs therapy in $57 \%$ and $74 \%$ of cases respectively [18]. In contrast with the Danish study [18] we observed that primary care physicians in the studied CCG were embracing the use of DOACs as $72 \%$ of new DOACs prescriptions were solely initiated by primary care prescribers. This finding also differs from prescribing trends seen in Canada [19] and USA [20], where secondary care physicians seem to drive the uptake of DOACs. Additionally, while in the studied CCG, between 2014 and beginning of 2015, DOACs were the predominant OAC initiated by primary care prescribers, in Canada it was warfarin. Further research should explore how embracing use of DOACs in primary care contributes to the overall uptake of DOACs.

The proportion of females initiated on OACs increased during the study period. This was not surprising and could be attributed to the change in the AF-related stroke risk calculation, moving from the $\mathrm{CHADS}_{2}$ to $\mathrm{CHA}_{2} \mathrm{DS}_{2}$-VASc score. This meant that female patients aged 65 and over were candidates for the OAC therapy [9]. The use of DOACs between sexes was similar, which supports the close to even split reported by Loo et al. (2017) in their study of DOAC prescribing in primary care in the UK [14]. However, with increasing uptake of DOACs in the UK, further studies could re-evaluate if DOAC use varies between sexes, and if so reasons behind it.

In this study, the limited evidence available suggests that when patients started on DOAC rather than warfarin, they were more likely to be involved in the decision-making, possibly due to being presented with different therapy choices. Previous evidence seems to corroborate that the uptake of DOACs increases if patients are involved in decisions about their anticoagulation therapy [21-23]. While a systematic review showed that patients who were not involved in these decisions were prescribed warfarin [23], two quantitative studies have shown that patients prescribed DOAC were more likely to be involved in the decision-making and presented with several therapy options [21,22]. It seems that patient involvement in the decision-making about their anticoagulation therapy may enable the grater uptake of DOACs. Therefore, this further strengthens why consultations should move from being paternalistic and involve patients in meaningful discussions about their treatment options.

To our knowledge, our study is the only study exploring on which OAC (warfarin or DOAC) the patient with prior aspirin monotherapy will be initiated. In this study, 140 patients were switched from aspirin to OAC therapy. This was expected as the use of antiplatelet monotherapy for stroke prevention has declined over years due to greater evidence for OAC use [9,15,24,25]. It is interesting that more patients were moved from therapy without monitoring (i.e. aspirin) to therapy requiring regular monitoring (i.e. warfarin) rather than without regular monitoring (i.e. DOAC). This may be due to lack of patient involvement in the decision-making as we have observed with warfarin initiation but requires further exploration.

\section{Conclusions}

This study has demonstrated that the early uptake of DOACs was embraced by primary care prescribers in this particular CCG, although findings cannot be generalised. Future, bigger studies with data from multiple CCGs will allow for an in-depth investigation of whether these findings are representative nationally, as well as providing a glimpse of reasons why the early uptake of DOACs may be higher in certain areas and lower in others. Future studies investigating the barriers and enablers to the uptake of DOAC in the UK will also provide understanding of how to increase uptake and promote optimal and rational use of these medicines in both primary and secondary care for reducing AF-related strokes. 
Uptake of oral anticoagulants for stroke prevention in atrial fibrillation

\section{Acknowledgements}

The authors would like to thank Dr Iuri Marques, from the University of Bradford, for his advice on statistical analysis in this manuscript.

\section{Compliance with Ethical Standards}

\section{Funding}

This study was funded by Bayer Pharmaceuticals via unrestricted educational grant. Bayer Pharmaceutical played no role in the design, conduct, analysis of this study, or writing of this manuscript.

\section{Ethical Approval}

Ethics approval was granted Chair of the Biomedical, Natural, Physical and Health Sciences Research Ethics Panel at the University of Bradford.

\section{Informed consent}

Informed consent is not required for this type of study.

\section{Conflict of interest}

Kristina Medlinskiene has received a sponsorship from Bayer to attend a conference (conference fee, accommodation, and travel expenses).

Matthew Fay has received funding from Bayer, Boehringer-Ingelheim, Bristol Myers Squibb, Dawn 4S, INRStar, Pfizer for travel expenses. His partnership (Westcliffe Health Innovations) has received funding from Bayer, Boehringer-Ingelheim, Bristol Myers Squibb, Dawn 4S, INRStar, Pfizer for my time for presentations of consultancy and Ad boards.

Duncan Petty has received has received honoraria for consultancy from Bayer and Pfizer. 


\section{References:}

1. Lane DA, Skjoth F, Lip GYH, LarsenTB, Kotecha D. Temporal trends in incidence, prevalence, and mortality of atrial fibrillation in primary care. J Am Heart Assoc. 2017;6:e005155.

2. Wolf PA, Abbott RD, Kannel WB. Atrial fibrillation as an independent risk factor for stroke: the Framingham study. Stroke. 1999;22:938-8.

3. Feigin VL, Norrving B, Mensah GA. Global burden of stroke. Circ Res. 2017;120:439-48.

4. Saka O, McGuire A, Wolfe, C. Cost of stroke in the United Kingdom. Age and Ageing. 2009;38:27-32.

5. Kirchhof P, Benussi S, Kotecha D, Ahlsson A, et al. ESC Guidelines for the management of atrial fibrillation developed in collaboration with EACTS. Eur Heart J. 2016;37:2893-2962.

6. Bai Y, Guo SD, Deng H, Shantsila A, et al. Effectiveness and safety of oral anticoagulants in older patients with atrial fibrillation: a systematic review and meta-regression analysis. Age Ageing. 2017;47:9-17.

7. Burn J, Pirmohamed M. Direct oral anticoagulants versus warfarin: is new always better than old? Open Heart. 2015;5:e000712.

8. The Association of the British Pharmaceutical Industry. One year on: Why are patients still having unnecessary AF-related strokes? 2016. http://www.abpi.org.uk/media/1404/safi_one_year_on.pdf Accessed 23 Apr 2018.

9. National Institute for Health and Care Excellence. Atrial Fibrillation: management (CG180). 2014. https://www.nice.org.uk/guidance/cg180/. Accessed 5 Dec 2016.

10. National Institute for Health and Care Excellence. Costing report: atrial fibrillation. Implementing the NICE guideline on atrial fibrillation (CG180). 2014. https://www.nice.org.uk/guidance/cg180/resources/costingreport-243730909. Accessed 30 May 2018

11. Camm AJ, Pinto FJ, Hankey GJ, Andreotti F, et al. Non-vitamin K antagonist oral anticoagulants and atrial fibrillation guidelines in practice: barriers to and strategies for optimal implementation. Europace. 2015;17:1007-17.

12. NHS Yorkshire and Humber Commissioning Support Group. West Yorkshire AC Clinic Provision. 2014. http://www.yhscn.nhs.uk/media/PDFs/cvd/Stroke/Documents_AF/yhscn-stroke-

10ccStrokeACClinicBaseline-20150825.pdf. Accessed 30 May 2018.

13. Potpara TS, Pison L, Larsen TB, et al. How are patients with atrial fibrillation approached and informed about their risk profile and available therapies in Europe? Results of the European Heart Rhythm Association Survey. Europace. 2015;17:468-72.

14. Loo SY, Dell'Aniello S, Huiart L, Renoux C. Trends in the prescription of novel oral anticoagulants in UK primary care. Br J Clin Pharmacol. 2017;2096-106.

15. Cowan JC, Wu J, Hall M, Orlowski A, West RM, Gale CP. A 10 year study of hospitalized atrial fibrillationrelated stroke in England and its association with uptake of oral anticoagulation. Eur Heart J. 2018;0:1-9.

16. NHS Employers website. Changes to QOF 2015/2016. 2016. http://www.nhsemployers.org/yourworkforce/primary-care-contacts/general-medical-services/quality-and-outcomes-framework/changes-toqof-2015-16. Accessed 7 Aug 2018.

17. Open Prescribing. Direct Oral Anticoagulants (DOACs) by all CCGs. https://openprescribing.net/measure/doacs/. Accessed 7 Aug 2018.

18. Pottegard A, Poulsen BK, Larsen MD, Hallas J. Dynamics of vitamin K antagonists and new oral anticoagulant use in atrial fibrillation: a Danish drug utilization study. J Thromb Haemost. 2014;12: $1413-8$.

19. Weitz JI, Semchuk W, Turpoie AG, et al. Trends in Prescribing Oral Anticoagulants in Canada, 2008-2014. Clin Ther. 2015;37:2506-14.

20. O’Neal WT, Sandesara PB, Claxton JS, et al. Provider speciality, anticoagulation prescription patterns, and stroke risk in atrial fibrillation. J Am Heart Assoc. 2018;7:e007943.

21. Andrade JG, Krahn AD, Skanes AC, Purdham D, Ciaccia A, Conors S. Value and preferences of physicians and patients with nonvalvular atrial fibrillation who receive oral anticoagulation therapy for stroke prevention. Can J Cardiol. 2017 32;747-753.

22. Choi JC, DiBonaventura M, Kopenhafer L, Nelson WW. Survey of the use of warfarin and the newer anticoagulant dabigatran in patients with atrial fibrillation. Patient Prefer Adherence. 2014 8;167-177.

23. Borg CX, Shaw R, Lane DA. Patients' and health professionals' views and experiences of atrial fibrillation and oral-anticoagulation therapy: a qualitative meta-synthesis. Patient Educ Couns. 2012 88;330-337.

24. Verheugt FWA, Gao H, Mahmeed WA, Ambrosio G, Anghchaisuksiri P, Atar D, Bassand JP, Camm AJ, Cools F, Eikelboom J, Kayani G, Lim TW, Misselwitz F, Pieper KS, van Eickels M, Kakkar AF, 
Uptake of oral anticoagulants for stroke prevention in atrial fibrillation

GARFIELD-AF Investigators. Characteristics of patients with atrial fibrillation prescribed antiplatelet monotherapy compared with those on anticoagulants: insights from the GARFIELD-AF registry. Eur Heart J. 2018;39:464-73.

25. Royal College of Physicians. Sentinel Stroke National Audit Programme (SSNAP). Clinical audit April-July 2017 public report. National results. Available from:

https://www.strokeaudit.org/Documents/National/Clinical/AprJul2017/AprJul2017-PublicReport.aspx Accessed 18 March 2018. 
1 Table 1.

2 Demographics of new patients initiated on oral anticoagulant therapy between 1/04/2012 and 31/03/2015.

\begin{tabular}{|c|c|c|c|c|c|c|c|c|c|}
\hline & \multicolumn{2}{|c|}{ 1/4/2012-31/3/2013 } & \multicolumn{2}{|c|}{ 1/4/2013-31/3/2014 } & \multicolumn{2}{|c|}{ 1/4/2014-31/3/2015 } & \multicolumn{3}{|c|}{ TOTAL } \\
\hline & $\begin{array}{l}\text { Initiated on } \\
\text { warfarin } \\
\mathrm{N}(\%)\end{array}$ & $\begin{array}{l}\text { Initiated on } \\
\text { DOACs } \\
\mathrm{N}(\%)\end{array}$ & $\begin{array}{l}\text { Initiated on } \\
\text { warfarin } \\
\mathrm{N}(\%)\end{array}$ & $\begin{array}{l}\text { Initiated on } \\
\text { DOACs } \\
\mathrm{N}(\%)\end{array}$ & $\begin{array}{l}\text { Initiated on } \\
\text { warfarin } \\
\mathrm{N}(\%)\end{array}$ & $\begin{array}{l}\text { Initiated on } \\
\text { DOACs } \\
\mathrm{N}(\%)\end{array}$ & $\begin{array}{l}\text { Initiated on } \\
\text { warfarin } \\
\mathrm{N}(\%)\end{array}$ & $\begin{array}{l}\text { Initiated on } \\
\text { DOACs } \\
\mathrm{N}(\%)\end{array}$ & $\begin{array}{l}\text { Initiated on } \\
\text { warfarin or DOAC } \\
\mathrm{N}(\%)\end{array}$ \\
\hline Total & 119 & 11 & 85 & 32 & 58 & 55 & 262 & 98 & 360 \\
\hline \multicolumn{10}{|c|}{ Age (years)* } \\
\hline Mean \pm SD & $77 \pm 11$ & $75 \pm 7$ & $76 \pm 9$ & $78 \pm 10$ & $72 \pm 12$ & $79 \pm 11$ & $76 \pm 11$ & $78 \pm 10$ & $76 \pm 11$ \\
\hline$<65$ & $16(13)$ & $2(18)$ & $12(14)$ & $2(6)$ & $15(26)$ & $6(11)$ & $43(16)$ & $10(10)$ & $53(15)$ \\
\hline $65-74$ & $26(22)$ & $1(9)$ & $26(31)$ & $6(19)$ & $14(24)$ & $12(22)$ & $66(25)$ & $19(19)$ & $85(24)$ \\
\hline $75-84$ & $46(39)$ & $8(73)$ & $32(38)$ & $14(44)$ & $20(35)$ & $19(34)$ & $98(37)$ & $41(42)$ & $139(39)$ \\
\hline $85-90$ & $21(18)$ & $0(0)$ & $11(13)$ & $8(25)$ & $6(10)$ & $7(13)$ & $38(15)$ & $15(15)$ & $53(15)$ \\
\hline$>90$ & $10(8)$ & $0(0)$ & $4(5)$ & $2(6)$ & $3(5)$ & $11(20)$ & $17(7)$ & $13(13)$ & $30(8)$ \\
\hline \multicolumn{10}{|l|}{ Sex } \\
\hline Male & $66(55)$ & $8(73)$ & $48(56)$ & $17(53)$ & $26(45)$ & $26(47)$ & $140(53)$ & $51(52)$ & $191(53)$ \\
\hline Female & $53(45)$ & $3(27)$ & $37(44)$ & $15(47)$ & $32(55)$ & $29(53)$ & $122(47)$ & $47(48)$ & $169(47)$ \\
\hline
\end{tabular}

$3 S D$ standard deviation, DOACs direct oral anticoagulants. 